\title{
Saúde materna em tempos de COVID-19: o que sabemos e para onde vamos?
}

\section{Maternal health in COVID-19 times: what we know and where are we going?}

\author{
Cássia Regina Gotler Medeiros ${ }^{1}$ \\ Carolina Dutra Degli Esposti ${ }^{2}$ \\ Katrini Guidolini Martinelli²
}

${ }^{1}$ Universidade do Vale do Taquari. Lajeado/RS, Brasil. ${ }^{2}$ Universidade Federal do Espírito Santo. Vitória/ES, Brasil.

Em janeiro de 2020 o mundo tomou conhecimento da existência de uma nova cepa de coronavírus, o SarsCoV-2 (Síndrome Respiratória Aguda Grave - coronavírus 2), que causa a doença denominada de COVID-19. Em 11 de março de 2020, a Organização Mundial da Saúde (OMS) elevou a COVID-19 à pandemia devido à disseminação geográfica alcançada. Em 21 de março de 2021 o número de casos de COVID-19 confirmados no mundo ultrapassava os 120 milhões e no Brasil, os 11 milhões. Já morreram mais de 2,7 milhões de pessoas no mundo e 290 mil pessoas no Brasil devido à infecção por Sars-CoV-2,2.

A COVID-19 pode causar complicações graves, principalmente em pessoas idosas e com comorbidades, mas todos podem ser afetados, incluindo mulheres grávidas e recém-nascidos. Não houve a indicação formal, por parte das autoridades sanitárias do Brasil, de inclusão das gestantes no grupo de risco, podendo garantir um afastamento protegido do trabalho. Esta iniciativa foi tomada individualmente apenas por algumas organizações.

Resultados negativos podem ser esperados em gestantes com sintomas de febre e tosse, especialmente naquelas que ficam hospitalizadas devido à gravidade dos sintomas ${ }^{3}$. Até o fim de fevereiro, do total de pessoas hospitalizadas desde o início da pandemia por Sars-CoV-2 ( $\mathrm{n}=709.404)$, as gestantes representavam $0,8 \%(\mathrm{n}=5.632)$ dos casos ${ }^{4,5}$.

Estudos iniciais não encontraram maior susceptibilidade das gestantes às complicações da COVID-19, entretanto, posteriormente, começaram a surgir casos de gestantes com doença grave e morte materna. Os principais sintomas apresentados pelas gestantes são tosse $(71,4 \%)$, febre $(63,3 \%)$ e dispneia $(34,4 \%)$, entretanto cerca de $15 \%$ delas são assintomáticas ${ }^{6}$. Quando as gestantes são sintomáticas, especialmente se os sintomas são graves, podem ser esperados resultados negativos ${ }^{3}$

Sintomas graves de COVID-19 na gestação mostraram-se associados à idade materna aumentada (OR: 1,83; IC95\%: 1,27-2,63), índice de massa corporal elevada (OR: 2,37; IC95\%: 1,83-3,07), comorbidade materna pré-existente 
(OR: 1,81; IC95\%: 1,49-2,20), hipertensão crônica (OR: 2,0; IC95\%: 1,14-3,48), diabetes prévia (OR: 2,12; IC95\%: 1,622,78) e pré-eclâmpsia (OR: 4,21; IC95\%: 1,27-14,0) .

De forma geral, a infecção por COVID-19 mostra-se associada à prematuridade espontânea (5\%), prematuridade por indicação obstétrica $(18,4 \%)^{6}$, mortalidade materna $(0,7 \%)$, admissão materna e neonatal na unidade de tratamento intensivo $(4,2 \%$ e $25,6 \%)$ e natimortalidade $(0,9 \%)^{7}$. Porém, as evidências científicas ainda são de baixa qualidade e as recomendações, em grande parte das vezes, baseiam-se na opinião de especialistas 8 .

Segundo o Royal College of Obstetricians and Gynaecologists (2021) ${ }^{8}$, Reino Unido, a evidência científica disponível até o momento para orientar os profissionais da saúde responsáveis pelo cuidado pré-natal mostram que mulheres grávidas com COVID-19, quando comparadas com mulheres grávidas sem COVID-19, têm maiores taxas de internação em unidades de tratamento intensivo, possivelmente mais relacionadas a regras severas para admissão, do que à presença de doença mais grave; o risco aumentado de morte relacionada COVID-19 não está totalmente esclarecido no mundo; e que as gestantes com COVID-19 que requerem hospitalização têm apresentado piores resultados maternos, incluindo risco de morte, apesar da baixa ocorrência. Aproximadamente 8 a $11 \%$ das gestantes e lactantes infectadas pelo Sars-CoV-2 necessitam de hospitalização e cerca de 2 a 5\% necessitam de atendimento em unidades de terapia intensiva?

Quanto aos óbitos no Brasil, a hipertensão e a obesidade em gestantes são fatores que contribuem para esse desfecho, pois a resposta inflamatória presente nessas situações pode levar a um pior prognóstico materno ${ }^{10}$. A té o fim de fevereiro, 303 gestantes foram à óbito por COVID-19 no Brasil, sendo, em sua maioria, pardas ( $\mathrm{n}=150 ; 49,5 \%$ ), na faixa etária de 30-39 anos ( $\mathrm{n}=144 ; 47,5 \%)$ e no terceiro trimestre gestacional $(\mathrm{n}=153 ; 54,5 \%)^{4,5}$. Mulheres no puerpério também foram significativamente acometidas? .

Ao verificar que mudanças fisiológicas na gestante estavam predispondo a infecções respiratórias graves, principalmente no puerpério, o Ministério da Saúde publicou a Nota Técnica 13, em maio de $2020^{11}$, recomendando testagem diagnóstica na internação de gestantes e, posteriormente, com a rápida deterioração nas condições clínicas de gestantes e puérperas infectadas, estabeleceu-se a testagem de gestantes cerca de 15 dias anteriores ao parto e na internação quando isto não fosse possível.

A preocupação também se estendeu à alta hospitalar, cuja recomendação, envolveu a ativação da Estratégia Saúde da Família/Unidade Básica de Saúde de referência, para o monitoramento sistemático da puérpera e do recémnascido neste período, especialmente os mais vulneráveis ${ }^{11}$.

Questionamentos foram surgindo com o avanço da pandemia no que diz respeito à conduta na atenção à saúde de gestantes e puérperas. Inicialmente, a preocupação concentrou-se em garantir a continuidade do pré-natal, a fim de evitar intercorrências por outras patologias. Mais tarde, constatou-se que muitas gestantes com COVID-19, orientadas a aguardarem em casa em caso de sintomas leves - como o restante da população - chegavam com a doença muito agravada ao hospital. Esta situação apresentou-se pior em locais de difícil acesso aos serviços de saúde, onde problemas crônicos da assistência à saúde da mulher, como recursos insuficientes, baixa qualidade pré-natal, insuficiência de leitos, disparidades raciais e violência obstétrica, podem ter contribuído para a razão de mortalidade materna por COVID-19 no Brasil ser uma das mais altas do mundo ${ }^{10,12}$.

Com relação à vacinação de gestantes contra a COVID-19, apesar de nenhuma das vacinas atualmente disponíveis ter sido submetida a ensaios clínicos específicos com mulheres grávidas, com a Nota Técnica no 1 , em março de $2021^{9}$, o Ministério da Saúde orienta que gestantes, puérperas e lactantes devem ser avaliadas e orientadas quanto ao risco de contágio e exposição, não havendo contraindicação da vacinação deste grupo com as vacinas utilizadas no Brasil até o momento. Recomenda-se que gestantes com comorbidades, pertencentes aos grupos prioritários definidos pelo próprio Ministério, sejam vacinadas contra a COVID-19, desde que possam tomar uma decisão esclarecida e que tenham sua vontade de vacinar-se ou não respeitada? .

Existem, portanto, muitas incertezas sobre a existência de maior risco de contágio em gestantes, de maior gravidade da doença nesse grupo ou de maiores complicações para a mãe e o bebê relacionadas à pandemia de COVID-19. 
Assim, ficam algumas reflexões sobre as orientações e decisões tomadas no Brasil em relação à proteção da vida de gestantes e recém-nascidos. Será que elas foram adequadas, levando em consideração o alto impacto do óbito materno nas famílias e no desenvolvimento da criança recém-nascida? A dignidade à vida dessas mulheres foi garantida, num momento em que medidas de isolamento social são requeridas, levando a mudanças econômicas e sociais em todo o mundo? Elas receberam apoio em suas inseguranças, tão comuns e possivelmente agravadas no contexto da pandemia? Como puderam essas mulheres manter sua saúde mental nesse período? Elas conseguiram?

A partir dessas inquietações e tantas outras possíveis, revela-se um terreno fértil às investigações científicas que abordem a gestação e puerpério no contexto da COVID-19. Vislumbra-se que muitos estudos possam e devam ser realizados, no sentido de revelar os detalhes dessa complexa realidade. Além disso, é preciso que os gestores e profissionais responsáveis pela atenção ao ciclo gravídico-puerperal busquem incessantemente as melhores evidências para balizar suas decisões e orientações quanto ao cuidado desse grupo nesse momento de pandemia.

Por último, as mulheres grávidas e suas famílias e redes de apoio precisam ter acesso aos serviços de saúde e fontes confiáveis de informação sempre que sentirem necessidade, para que este seja adequado e realizado em tempo oportuno. Precisam, ainda, manter-se em segurança e expostas ao menor risco possível à doença, buscando o apoio das autoridades sanitárias quando necessário. Estas são algumas recomendações que objetivam manter uma saúde materna adequada em tempos de COVID-19.

\section{REFERÊNCIAS |}

1. Organização Mundial da Saúde (OMS), Organização Pan-americana da saúde (OPAS). Folha informativa - COVID-19 (doença causada pelo novo coronavírus) 2021.

2. WHO. World Health Organization. WHO Coronavirus (COVID-19) Dashboard. Disponível em: https:/ covid19.who. int/. Acessado em: 21.03.2021.

3. Papapanou M, Papaioannou M, Petta A, Routsi E, Farmaki M, Vlahos N, et al. Maternal and Neonatal Characteristics and Outcomes of COVID-19 in Pregnancy: An Overview of Systematic Reviews. Int. J. Environ. Res. Public Health 2021, 18, 596. https://doi.org/10.3390/ijerph18020596.

4. Boletim Epidemiológico Especial. COE-COVID19. Brasília (DF): Ministério da Saúde. Secretaria de Vigilância em Saúde; 2020:44.

5. Boletim Epidemiológico Especial. COE-COVID19. Brasília (DF): Ministério da Saúde. Secretaria de Vigilância em Saúde; 2021:52.

6. Khalil A, Kalafat E, Benlioglu C, O’Brien P, Morris E, Draycott T, et al. SARS-CoV-2 infection in pregnancy: A systematic review and meta-analysis of clinical features and pregnancy outcomes. E Clinical Medicine 2020, 25, 100446. https://doi.org/10.1016/j.eclinm.2020.100446.

7. Allotey J, Stallings E, Bonet M, Yap M, Chatterjee S, Kew T, et al. Clinical manifestations, risk factors, and maternal and perinatal outcomes of coronavirus disease 2019 in pregnancy: Living systematic review and meta-analysis. BMJ 2020, 370, m3320. http://dx.doi.org/10.1136/bmj.m3320.

8. Royal College of Obstetricians and Gynaecologists. Coronavirus (COVID-19) Infection in Pregnancy: Information for healthcare professionals. Version 13: Published Friday 19 February 2021. Disponível em: https://www.rcog.org.uk/ globalassets/documents/guidelines/2021-02-19-coronavirus-covid-19-infection-in-pregnancy-v13.pdf. Acessado em 21.03.2021.

9. Secretaria de Atenção Primária à Saúde. Nota Técnica no 1/2021 - DAPES/SAPS/MS. Brasília: Ministério da Saúde. 2021. 
10. Nakamura-Pereira M, Amorim MMR, Pacagnella RC, Takemoto MLS, Penso FCC, Rezende-Filho J, et al. COVID-19 and Maternal Death in Brazil: An Invisible Tragedy. Rev Bras Ginecol Obstet. 2020;42(8):445-7.

11. Secretaria de Atenção Primária à Saúde. Nota Técnica no 13/2020 - DAPES/SAPS/MS. Brasília: Ministério da Saúde. 2020.

12. Souza ASR, Amorim MMR. Mortalidade materna pela COVID-19 no Brasil. Rev. Bras. Saúde Matern. Infant. 2021; 21(Supl. 1): S257-S261. 